\title{
Daily intake of probiotic strain Bacillus subtilis DE111 supports a healthy microbiome in children attending day-care
}

\author{
A. Paytuví-Gallart ${ }^{1}$, W. Sanseverino ${ }^{1}$ and A.M. Winger ${ }^{2 *}$ \\ ${ }^{1}$ Sequentia Biotech SL, Carrer Comte d'Urgell 240, Barcelona, Spain; ${ }^{2}$ Deerland Probiotics and Enzymes, UCC BioInnovation \\ Unit, 1 College Road, Cork, Ireland; awinger@deerland.com
}

Received: 14 February 2020 / Accepted: 24 June 2020

(c) 2020 Wageningen Academic Publishers

OPEN ACCESS @(1)(0 RESEARCH ARTICLE

\begin{abstract}
There is ample evidence suggesting that modulations in gut microbiota play an important role in inflammation and immunity. In particular, the microbiota of children is highly susceptible to environment influences, such as infections. Consequently, probiotics and their ability to promote and support a healthy microbiome have been increasingly studied. This study aimed at investigating the effects of a probiotic supplement (Bacillus subtilis DE111) on the microbiome composition of preschool aged children attending day care. Healthy children aged 2-6 years old were randomised to receive either probiotic or placebo once a day for 8 weeks. No significant changes of the overall microbiome equilibrium were seen in between the two groups or from baseline to week 8 . However, alpha diversity was increased in the probiotic group from baseline to week $8(P<0.05)$, with no change in the placebo group. A decrease in the Firmicutes/Bacteroidetes ratio following probiotic supplementation $(P<0.05)$ was also observed. Differential abundance analysis revealed an increase in Alistepes $(P<0.01)$, Bacteroides $(P<0.05)$, Parabacteroides $(P<0.01)$, Odoribacter $(P<0.001)$ and Rikenellaceae $(P<0.001)$ in the probiotic group, most of which are involved in inflammation reduction. In addition, a decrease in Eisenbergiella $(P<0.001)$, Lactobacillales $(P<0.01)$ and Streptococcaceae $(P<0.01)$, which is considered pro-inflammatory, were also observed in the probiotic group. Together with a reduction of the F/B ratio observed in the probiotic group, these results suggest probiotic supplementation with Bacillus subtilis DE111 introduce subtle but positive changes in the microbiome of children aged 2-6 years old.
\end{abstract}

Keywords: Bacillus subtilis DE111, gut microbiome, paediatric population

\section{Introduction}

The number of children who attend day care centres in Europe is approximately $34 \%$ for $0-3$ year olds and $95 \%$ for 4-6 year olds (typically attending preschool) (Eurydice Report, 2019). It has been shown that children attending day care centres typically have an increased risk of developing gastrointestinal infections compared to children cared for at home, particularly during the winter season, often leading to the need for antibiotics (Enserink et al., 2013; Louhiala, 1997; Nafstad, 1999). Incidences of such infections warranting a general practitioner visit have been shown to be twice as prevalent compared to the general population of the same age (Enserink et al., 2013), with the most frequent infections being gastroenteritis (diarrhoea and vomiting) and flu-like illnesses (Enserink et al., 2013). Besides the obvious impact of these infections on the child, there are also additional social and economic consequences, such as parent work absences and higher medical expenses (Van der Wielen, 2010). There is a push globally to minimise the use of antibiotics in the population, therefore ways to support the health of children attending day care is of great interest.

Defined as 'live microorganisms which when administered in adequate amounts confer a health benefit on the host' (Hill et al., 2014) probiotics have the potential to support the maintenance of health in the population. A number of studies have been carried out in paediatric populations investigating the efficacy and microbiome modulatory potential of various strains of probiotics, primarily Lactobacillus, Bifidobacterium and Saccharomyces (El Manouni El Hassani et al., 2019; Laursen et al., 2017; 
Wallace, 2009; Xiao et al., 2019). These studies report mixed results and it is generally acknowledged that the effects of probiotics in the maintenance of health and ability to impact the microbiome is strain and dose dependent (Hojsak, 2017; Laursen and Hojsak, 2018). Interestingly, the microbiome of new-borns, infants (1-3 years old), adults (18-65 years old) and elderly (65 years old or older) has been extensively studied and reveals differences in microbial profiles throughout the aging process. However, to date, little is known about the gut microbial profile of older children (3 years and upwards) as there are comparatively fewer studies (Derrien et al., 2019).

While the primary probiotics studied and on the market are Lactobacillus, Bifidobacterium and Saccharomyces, there is an increasing interest and evidence in the use of Bacillus species as safe and effective probiotics (AnayaLoyola et al., 2019; Labellarte et al., 2015; Lefevre et al., 2017; Suva et al., 2016). Bacillus are spore-forming bacteria and confer many advantages over other probiotics. In their spore form, they can resist the harsh digestive environment, reaching and vegetating in the gut, thus supporting a healthy gastrointestinal (GI) tract. They have a long history of safe use in foods, with centuries of use of these bacteria for preparation of alkaline-fermented foods and soy-based food products (Inatsu et al., 2006; Ray et al., 2000; Wang and Fung, 1996). Additionally, Bacillus species have been found in the normal microflora of the gut in healthy adults (Hong et al., 2009) and children (Ellis-Pegler et al., 1975).

Bacillus species shown to have probiotic potential include Bacillus subtilis, Bacillus coagulans, Bacillus lichenoformis and Bacillus clausii (Cuentas et al., 2017; Horosheva et al., 2014; Lakshmi et al., 2017). B. subtilis DE111 is a probiotic strain of Bacillus that has been shown to support healthy gut function and promote digestive health in adults (Cuentas et al., 2017; Labellarte et al., 2015; Toohey et al., in press). It has also been shown to be safe for use in paediatric populations and promotes gastrointestinal health in day care-attending children (unpublished data). The aim of this study was to investigate the effect of daily intake of B. subtilis DE111 on the gut microbiome in day careattending children aged 2-6 years old.

\section{Materials and methods}

\section{Study design}

The current study was performed on stool samples obtained from a wider study in which safety/tolerance (adverse events) and impact on gastrointestinal and respiratory infections in preschool aged children was performed. This randomised, parallel, double-blind, placebo-controlled study was carried out between April and July 2019 in five paediatric centres in Slovenia. A flowchart of the study flow is depicted in Figure 1. The protocol was approved by the
National Medical Ethics Committee (Ministry of Health, Slovenia, 0120-569/2018/4) in Slovenia and registered on clinicaltrials.gov (NCT04077034). Each parent or legal guardian signed an informed consent form prior to enrolment in the study. Preschool children, aged 2-6 years were screened by their paediatricians and 102 children were recruited on the basis of inclusion (generally healthy, attending day-care) and exclusion (regular medication, immunodeficiency or severe chronic illness, low birth body weight $(<2,500 \mathrm{~g})$, low gestational age $(<37$ weeks)) criteria. Following a 4-week run-in period, 102 day-care attending children aged 2-6 years old received B. subtilis DE111 $\left(1 \times 10^{9} \mathrm{cfu}\right)$ or placebo once a day at home at breakfast for 8 weeks. Stools samples were collected at baseline and again at 8 weeks.

\section{Study product}

The active product comprised of the probiotic strain B. subtilis DE111 ( $1 \times 10^{9} \mathrm{cfu} /$ dose $)$, dextrose, tapioca maltodextrin, natural flavour and l-leucine. The placebo consisted of the same without the probiotic. Both products were packaged into individual $1.4 \mathrm{~g}$ stick packs and consumed either alone or with food or drink at breakfast.

\section{Stool collection}

Parents or caregivers of all participants were asked to collect a faecal sample in a sterile container (CLEARline cryogene tubes; Biosigma, Cona, Italy) at baseline and again at the end of the intervention. Faecal samples were stored in the

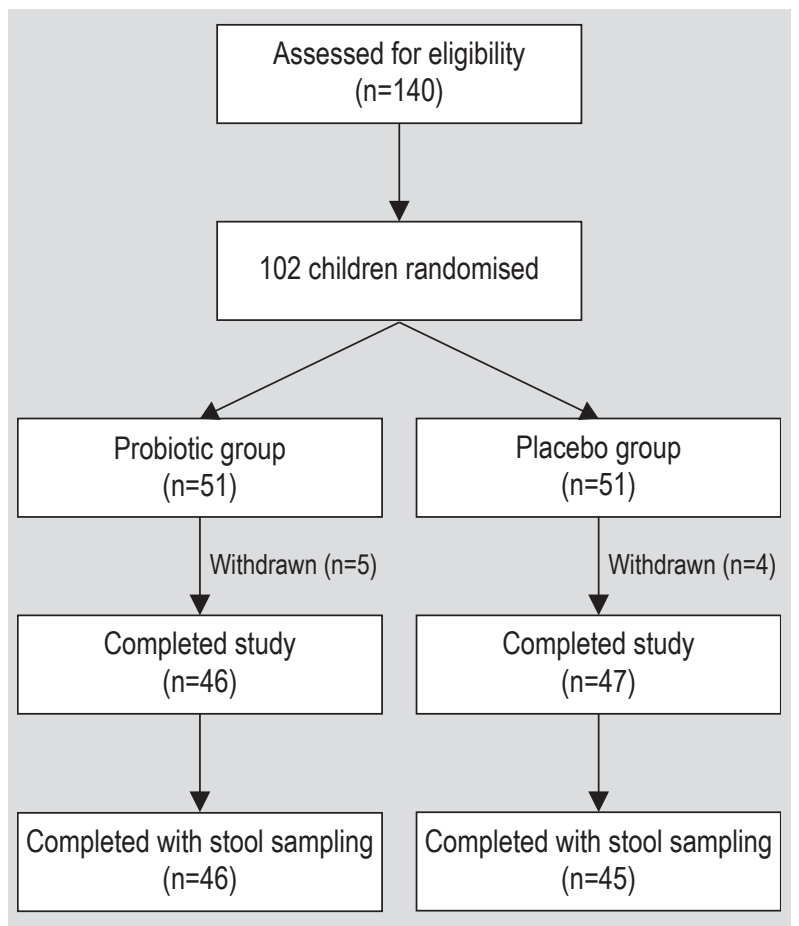

Figure 1. Flowchart of study design. 
freezer $\left(-20^{\circ} \mathrm{C}\right)$ at home immediately following collection. Frozen samples were transferred to the investigator sites and stored at $-20^{\circ} \mathrm{C}$ until transferring to $-80^{\circ} \mathrm{C}$ at the end of the intervention until DNA extraction.

\section{DNA extraction/16S sequencing}

DNA was extracted using a DNeasy PowerSoil Kit (Qiagen, Hilden, Germany) according to the manufacturer's instructions. The extracted DNA was quantified using Quant-IT PicoGreen (Invitrogen, Carlsbad, CA, USA). Input gDNA (12.5 ng) for each sample was amplified with 16S V3-V4 primers 16SF TCGTCGGCAGCGTCAGAT GTGTATAAGAGACAGCCTACGGGNGGCWGCAG and 16SR GTCTCGTGGGCTCGGAGATGTGTATAAGA GACAGGACTACHVGGGTATCTAATCC. A subsequent limited-cycle amplification step was performed to add multiplexing indices and Illumina sequencing adapters. The final products were normalised and pooled using the PicoGreen, and the size of libraries were verified using the LabChip GX HT DNA High Sensitivity Kit (PerkinElmer, Waltham, MA, USA). Sequencing was performed using the MiSeq $^{\text {Tim }}$ platform (Illumina, San Diego, CA, USA).

\section{Statistical analysis}

Raw reads were analysed with GAIA (v 2.02) (https:// metagenomics.sequentiabiotech.com) (Paytuví et al., 2019) to obtain operational taxonomic unit (OTU) tables at different taxonomic levels with their corresponding Shannon alpha-diversity and Bray-Curtis beta-diversity values were computed with the $\mathrm{R}$ package phyloseq (McMurdie and Holmes, 2013). Principal Coordinates Analysis ( $\mathrm{PCoA}$ ) was also performed using the function plot_ordination from the R package phyloseq. In order to see whether PCoA clusters significantly differed between experimental conditions, a permutational ANOVA (PERMANOVA) analysis using the $\mathrm{R}$ package adonis was carried out. DESeq2 (v 1.26) (Love et al., 2014) with default parameters was used to carry out a paired analysis between baseline and 8 week samples in both placebo and probiotic groups; significance threshold was set at $P$-adjusted 0.05 . A series of paired Wilcoxon tests (R package ggpubr) together with ggplot2, which was used for plotting purposes, were also performed to compare taxa and alpha-diversity indexes between baseline and 8 -week samples in both placebo and probiotic groups. Significance threshold was set at 0.05 . Differential analysis in biodiversity based on Hill numbers was performed using the method of Pallmann et al. (2012) implementation (R package mcpHill).

\section{Results and discussion}

\section{Baseline characteristics of participants}

Of the participants that completed the study and provided stool samples, a total of 42 males and 49 females were randomised to placebo or probiotic groups (Figure 1). There were no statistical differences in gender, age, height, weight, heart rate, respiratory rate or body temperature characteristics between groups (Table 1).

\section{Microbiome composition}

Analysis was performed on both placebo and probiotic arms at baseline and at the end of the trial. To obtain an overview of the microbiome composition for each analysed group, reads from same-group samples were summed and the percentage computed. The most abundant genera across all sample groups was an unknown genus belonging to the order Clostridiales, unknown Clostridiales (o), followed by Bacteroides, Bifidobacterium and Akkermansia (Figure 2).

When looking at individual samples scattered on a Principal Coordinates Analysis (PCoA) from Bray-Curtis dissimilarity values, it could be seen that samples tended to cluster with those having similar taxonomic profile (e.g. same mostabundant genera) (Figure 3A). Should probiotic intake alter the overall microbiome composition in the same direction, probiotic/week-8 samples would be expected to populate a specific location in the PCoA. However, no clear cluster was observed in the different groups: probiotic/baseline, probiotic/week-8, placebo/baseline, placebo/week-8 (PERMANOVA test, $P>0.05$ ). Bray-Curtis dissimilarity values for the same individual between baseline and week-8 was also analysed (Figure 3B). The average value for the placebo and probiotic group was 0.385 and 0.3824 , respectively, with no statistical difference observed

Table 1. Baseline characteristics of subjects. ${ }^{1}$

$\begin{array}{lll}\text { Characteristics } & \text { Placebo }(\mathrm{n}=\mathbf{4 5}) & \text { Active }(\mathrm{n}=\mathbf{4 6}) \\ \text { Gender } & & \\ \quad \text { Female } & 60 \%(27) & 48 \%(22) \\ \quad \text { Male } & 40 \%(18) & 52 \%(24) \\ \text { Age (months) } & 53.4 \pm 13.3 & 54.3 \pm 13.4 \\ \text { Height }(\mathrm{cm}) & 106.8 \pm 8.1 & 108.6 \pm 9.1 \\ \text { Weight }(\mathrm{kg}) & 18.2 \pm 3.1 & 19.5 \pm 3.8 \\ \text { Heart rate (beats/min) } & 100 \pm 10.9 & 97.8 \pm 13.6 \\ \text { Respiratory rate (breaths/min) } & 27.1 \pm 6.5 & 25.6 \pm 7.1 \\ \left.\text { Body temperature ( }{ }^{\circ} \mathrm{C}\right) & 36.6 \pm 0.3 & 36.7 \pm 0.3 \\ & \\ & \\ & \end{array}$




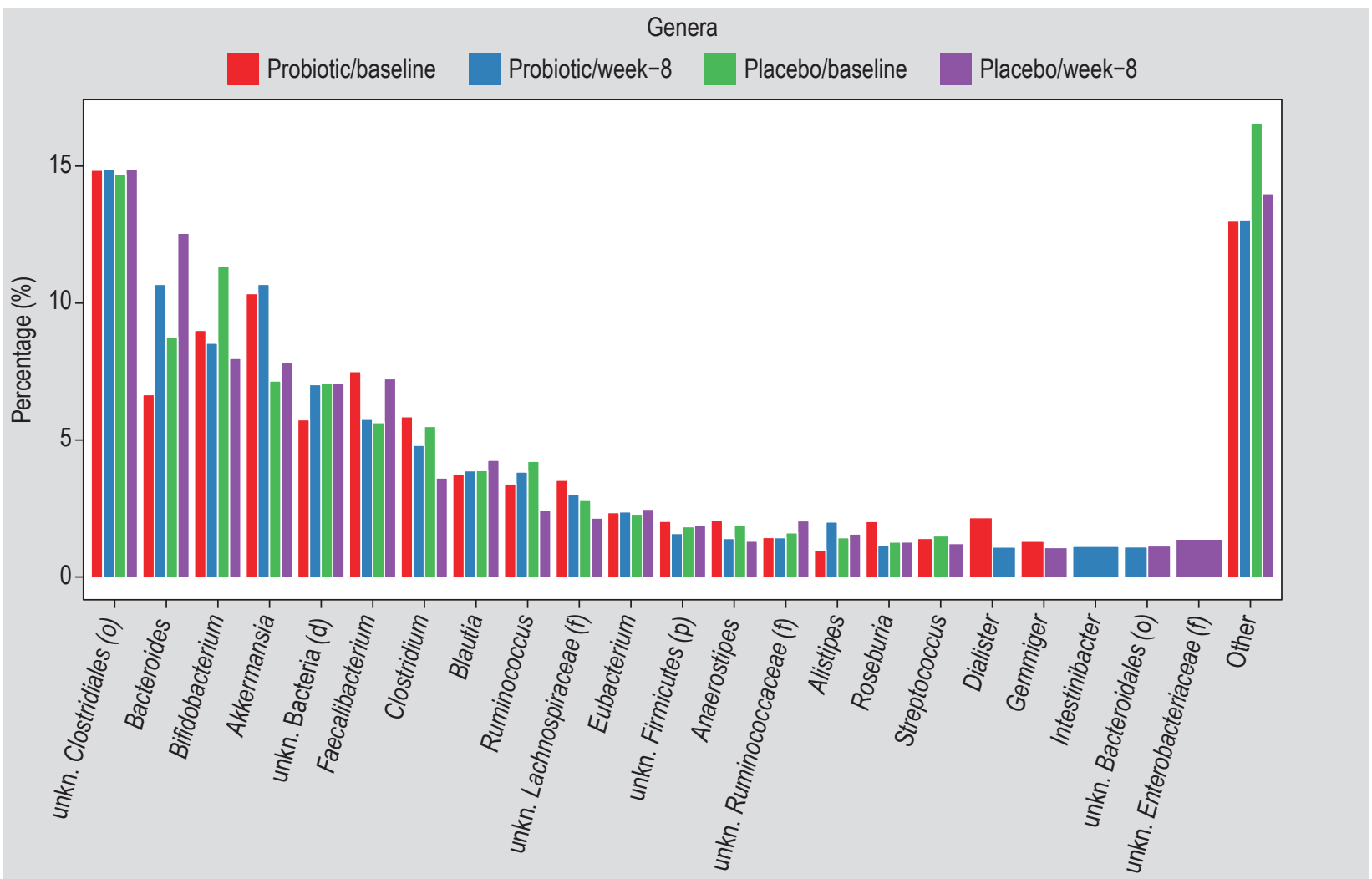

Figure 2. Taxonomic composition of probiotic and placebo groups. Genera with an abundance on average lower than $1 \%$ are classified to the group 'Other'.

(Wilcoxon test, $P>0.05$ ). Therefore, the overall taxonomic variation in each individual between baseline and week-8 was very similar in both placebo and probiotic groups. Taken together, these results suggest probiotic intake does not alter or perturb the overall microbiome equilibrium.

\section{Alpha-diversity analysis}

Alpha-diversity indices (richness, Shannon and Simpson indexes) were compared between the baseline and 8-week samples by means of paired Wilcoxon tests. At phylum level it could be seen that the intake of B. subtilis DE111 significantly increased $(P<0.05)$ both Shannon and Simpson alpha-diversity indexes (Figure 4). However, no change in the richness was observed between the baseline and 8 -week samples (Wilcoxon test, $P>0.05$ ). These results were confirmed by assessing differences in biodiversity using the Hill numbers under the Pallmann et al. (2012) implementation; we only obtained significant results in the probiotic group after $B$. subtilis DE111 intake for the Hill numbers q $1(P<0.05)$ and q $2(P<0.02)$.

Shannon and Simpson alpha-diversity indexes account for both richness (how many taxa) and evenness (how evenly these taxa are distributed) in a given sample, although evenness (especially in the Simpson index) contributes three times more than richness in these indexes (Reese and
Dunn, 2018; Strong, 2016). As an increase in the richness was not observed, the results indicate evenness is the factor that is contributing to the alpha diversity increase. This suggests that phyla are more evenly distributed from baseline to week 8 following probiotic supplementation. The gut microbiome contains microorganisms with different types of enzymes that are able to degrade or digest different molecules (e.g. plant polysaccharides). While a low evenness (few species with high abundance) would indicate limited enzyme diversity, a higher evenness would suggest a greater enzyme diversity, with additional functionalities adding to the metabolic activity of the gut microbiome. In this way, the increase in evenness following B. subtilis DE111 intake may in turn reflect an increase in the functional potential of the microbiome, which is typically considered beneficial for the individual (Everard et al., 2014). This hypothesis could be investigated further by additional data analyses, including functional information.

\section{Differential abundance analysis}

DESeq2 identified nine differential abundant taxa (FDR $<0.05)$ at genus level, with six taxa significantly increased after probiotic intake and three taxa significantly decreased. Among the increased taxa, all genera belonged to the phylum Bacteroidetes and included Alistipes, an unknown Bacteroidaceae genus, Odoribacter, Parabacteroides and 
A

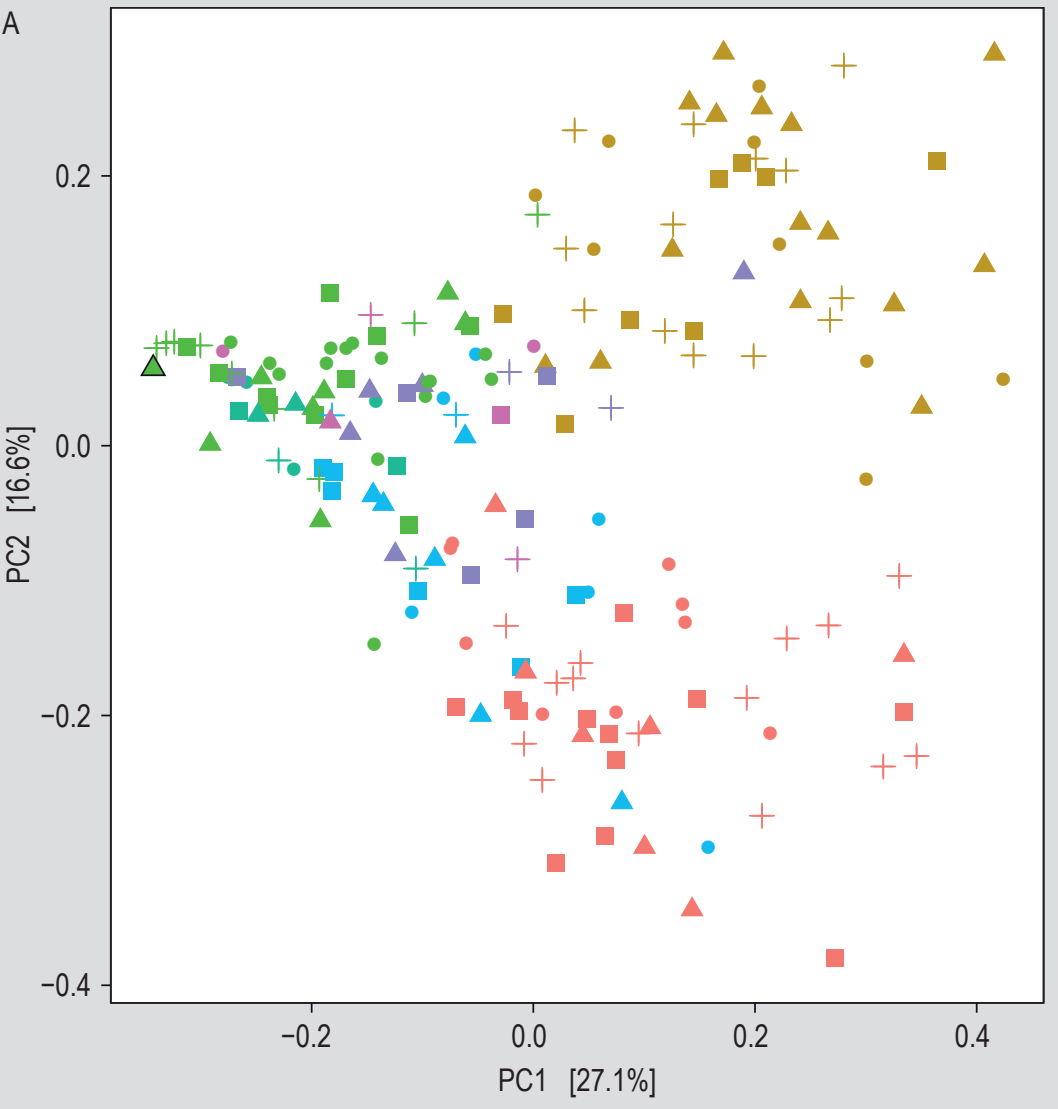

Group

- Placebo/Baseline

- Placebo/Week-8

- Probiotic/Baseline

+ Probiotic/Week-8

Most abundant

- Akkermansia

- Bacteroides

- Bifidobacterium

- Clostridium

- Faecalibacterium

- Other

- Ruminococcus

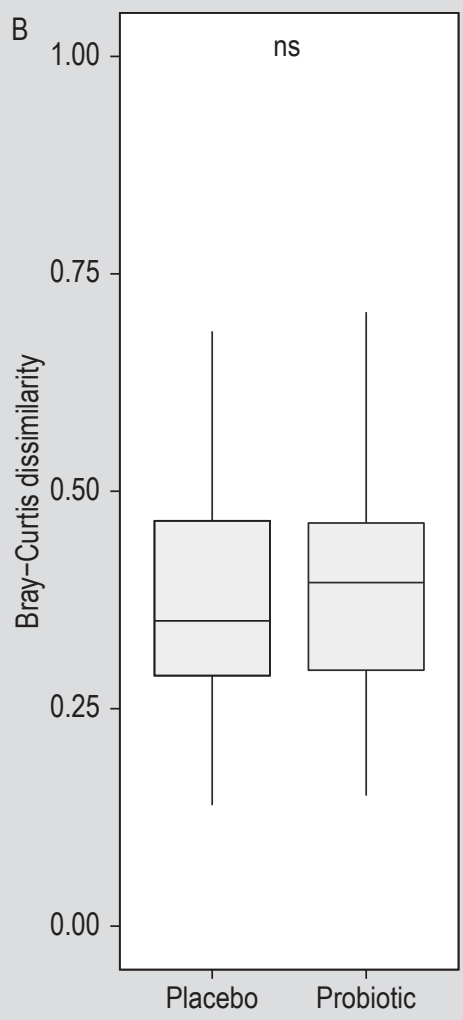

Figure 3. Beta-diversity analysis overview. (A) Clustering of samples by PCoA from Bray-Curtis dissimilarities. Unknown genera are not included. Genera that were most abundant in only three or less samples were grouped in 'Other'. (B) Boxplot assessing Bray-Curtis dissimilarities for each individual between baseline and week-8. Significance was assessed by means of paired Wilcoxon test. $\mathrm{ns}=P>0.05$. 


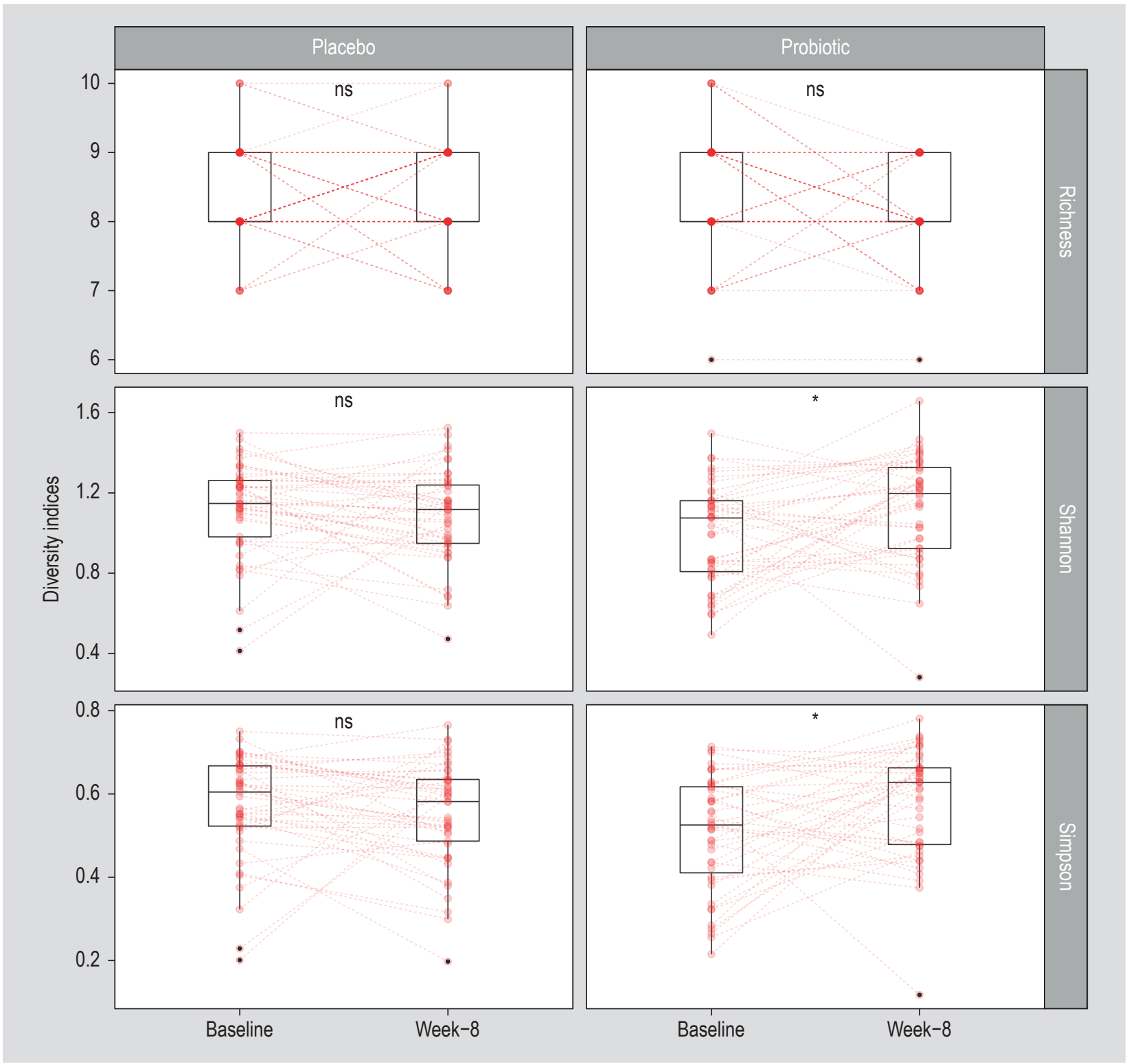

Figure 4. Alpha-diversity boxplots for richness, Shannon and Simpson indices. Same-patient samples are connected with dotted lines. Significance was assessed by means of paired Wilcoxon test. $P<0.05\left(^{*}\right) ; P>0.05$ (ns).

an unknown Rikenellaceae genus. Among the decreased taxa, all genera belonged to the phylum Firmicutes and included Eisenbergiella, an unknown Lactobacillales genus and an unknown Streptococcaceae genus (Figure $5 \mathrm{~A})$. Wilcoxon tests also identified that the abundance of genera Alistipes, Bacteroides, Eisenbergiella, Odoribacter and Parabacteroides were significantly altered (Figure $5 \mathrm{~B})$. In the placebo arm, only one differentially abundant genus (Pantoea) was identified by DESeq2 from baseline to week 8 . In this case, the level increased following the intervention. Since all significantly increased genera belong to Bacteroidetes and all significantly decreased bacteria belong to Firmicutes after probiotic treatment, possible changes in the Firmicutes/Bacteroidetes (F/B) ratio between groups was evaluated. Consistent with the results obtained from the differential abundance analysis, a significant decrease in the $\mathrm{F} / \mathrm{B}$ ratio after probiotic treatment was observed (Wilcoxon test, $P<0.05$ ) (Figure $5 \mathrm{C}$ ).

Bacteroides has been shown to play a role in reduction of inflammation, where lower levels of Bacteroides have been found in people suffering from inflammatory bowel disease (Zhou and Zhi, 2016). In a similar study, patients being administered with a Bacteroides thetaiotaomicron probiotic showed a significant reduction in colon inflammation (Delday et al., 2019). Like Bacteroides, Alistipes and Odoribacter are short-chain fatty acid (SCFA) producers. SCFAs are produced from fermentation of non-digestible dietary fibres, and includes propionate, acetate and butyrate. These molecules have an important role in the energy 
A
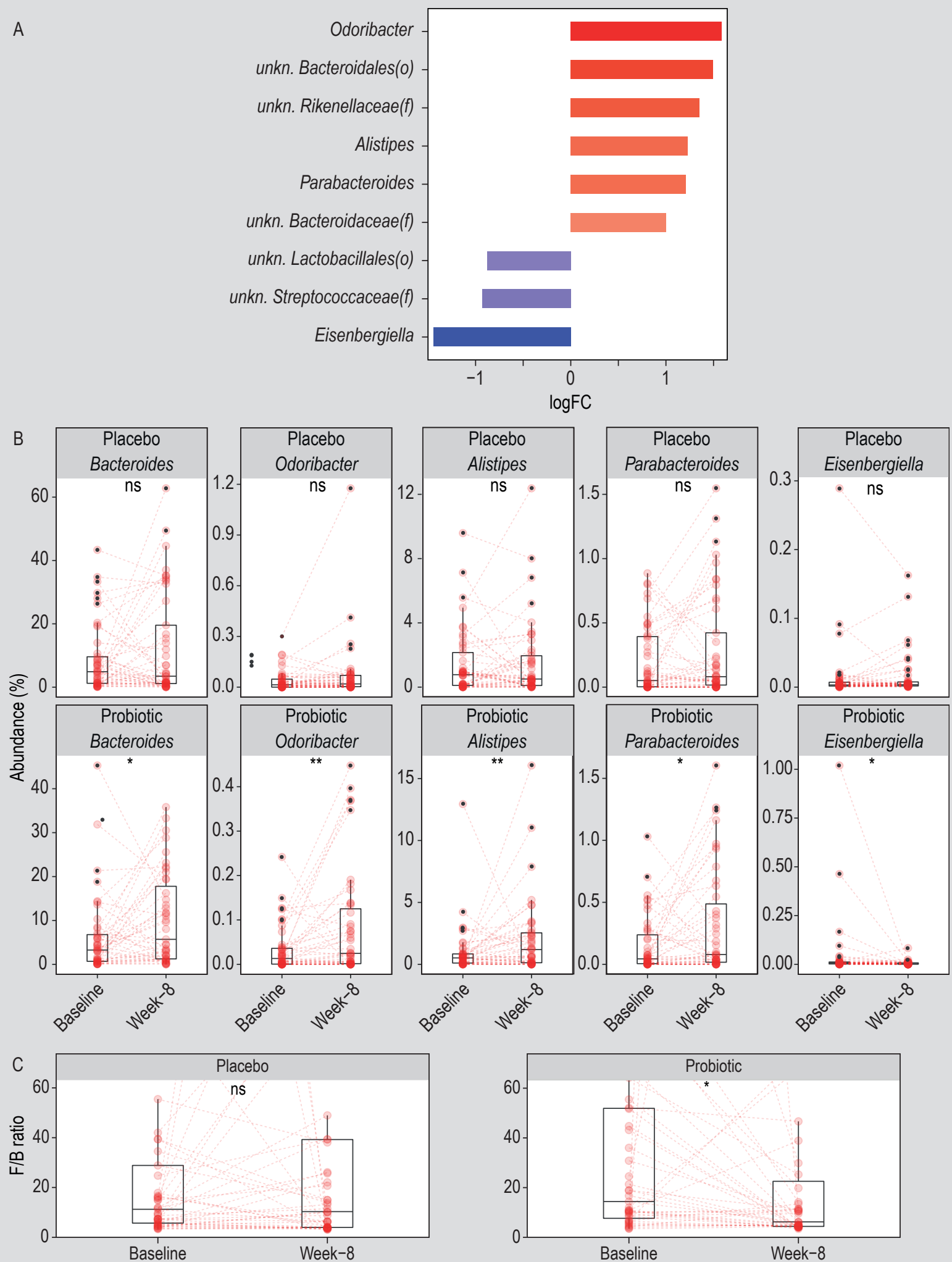

Figure 5. Differential abundance analysis overview. (A) Bar plot showing the log2 fold change (FC) of significant genera from DESeq2 analysis in the probiotic group. (B) Box plots showing the abundance of significant genera, by means of paired Wilcoxon tests, between baseline and 8-week samples in the probiotic and placebo groups. (C) Firmicutes to Bacteroidetes (F/B) ratio for placebo and probiotic groups between baseline and 8-week samples. $P \leq 0.01$ (**); $P \leq 0.05\left(^{*}\right) ; P>0.05$ (ns). 
homeostasis of the body (Byrne et al., 2015). Additionally, SCFAs are also implicated in immune regulation and inflammation where several studies have described a reduction of SCFAs producers in Inflammatory Bowel Diseases (IBD) (Forbes et al., 2016; Parada Venegas et al., 2019). In contrast, higher levels of Streptococcaceae have been shown to be associated with inflammation (Zeng et al., 2016) and increased susceptibility to diarrhoea in children (Hakim et al., 2018). Interestingly, in this study, a decrease in an unknown Lactobacillales was also observed in the probiotic arm. This order encompasses Streptococcaceae, however also includes Lactobacillus, strains of which have been shown to reduce the incidence of diarrhoea, albeit on a strain-specific level (Hojsak, 2017). Further studies, exploring a deeper analysis of the microbiome, to gain an understanding at a species level, would assist in further elucidating exactly what species are showing differential expression.

In addition to individual genera, the overall $\mathrm{F} / \mathrm{B}$ ratio has been extensively studied, with an increased F/B ratio correlating with increased prevalence of obesity and diabetes (Castaner et al., 2018). The association between the $\mathrm{F} / \mathrm{B}$ ratio and the immune system has also been assessed, where an increase in the $\mathrm{F} / \mathrm{B}$ ratio negatively correlated with immunoglobulin G and M levels (Shen et al., 2018). Overall, the results from this current study suggest that B. subtilis DE111 intake has an impact on the microbiome, supporting the growth of microbes involved in inflammation reduction and suppressing the growth of genera implicated in inflammation and GI disturbances. Future studies looking deeper within the microbiome to a species level, and indeed at the metabolome, will allow insight into the potential mechanisms of inflammation and GI support of this probiotic.

\section{Conclusions}

The current study showed that daily intake of the probiotic strain B. subtilis DE111 was able to introduce subtle but positive changes in the gut microbiome profile in children, aged 2-6 years old and attending day-care, without changing the overall microbiome equilibrium. An increase in alphadiversity at the phylum level was observed, suggesting an increased functional diversity of the microbiome of the probiotic group. Nine differentially abundant taxa at the genus level were observed in the probiotic group, six increased in abundance while three decreased. The six taxa increased in abundance were genera implicated in immune regulation and reduction of inflammation. A decrease in the $\mathrm{F} / \mathrm{B}$ ratio of the microbiome in children taking the probiotic supplement was also observed. Overall, these results suggest $B$. subtilis DE111 supports the maintenance of a healthy gut through subtle yet statistically significant modulations of the microbiome in preschool aged children.

\section{Conflict of interest}

This study was sponsored by Deerland Probiotics and Enzymes. AW Winger is an employee of Deerland Probiotics and Enzymes, but was not involved in the study conduct, data management or statistical analysis.

\section{References}

Anaya-Loyola, M.A., Enciso-Moreno, J.A., Lopez-Ramos, J.E., GarciaMarin, G., Orozco Alvarez, M.Y., Vega-Garcia, A.M., Mosqueda, J., Garcia-Gutierrez, D.G., Keller, D. and Perez-Ramirez, I.F., 2019. Bacillus coagulans GBI-30, 6068 decreases upper respiratory and gastrointestinal tract symptoms in healthy Mexican scholaraged children by modulating immune-related proteins. Food Research International 125: 108567. https://doi.org/10.1016/j. foodres.2019.108567

Byrne, C.S., Chambers, E.S., Morrison, D.J. and Frost, G., 2015. The role of short chain fatty acids in appetite regulation and energy homeostasis. International Journal of Obesity 39: 1331-1338. https:// doi.org/10.1038/ijo.2015.84

Castaner, O., Goday, A., Park, Y.-M., Lee, S.-H., Magkos, F., Shiow, S.-A.T.E. and Schroder, H., 2018. The gut microbiome profile in obesity: a systematic review. International Journal of Endocrinology 2018: 9. https://doi.org/10.1155/2018/4095789

Cuentas, A.M., Deaton, J., Khan, S., Davidson, J. and Ardita, C., 2017. The effect of Bacillus subtilis DE111 on the daily bowel movement profile for people with occasional gastrointestinal irregularity. Journal of Probiotics and Health 5: 189. https://doi. org/10.4172/2329-8901.1000189

Delday, M., Mulder, I., Logan, E.T. and Grant, G., 2019. Bacteroides thetaiotaomicron ameliorates colon inflammation in preclinical models of Crohn's disease. Inflammatory Bowel Diseases 25: 85-96. https://doi.org/10.1093/ibd/izy281

Derrien, M., Alvarez, A.-S. and De Vos, W.M., 2019. The gut microbiota in the first decade of life. Trends in Microbiology 27: 997-1010. https://doi.org/10.1016/j.tim.2019.08.001

El Manouni El Hassani, S., De Boer, N.K.H., Jansen, F.M., Benninga, M.A., Budding, A.E. and De Meij, T.G.J., 2019. Effect of daily intake of Lactobacillus casei on microbial diversity and dynamics in a healthy pediatric population. Current Microbiology 76: 1020-1027. https://doi.org/10.1007/s00284-019-01713-9

Ellis-Pegler, R.B., Crabtree, C. and Lambert, H.P., 1975. The faecal flora of children in the United Kingdom. Journal of Hygiene 75: 135-142. https://doi.org/10.1017/s002217240004715x

Enserink, R., Ypma, R., Donker, G.A., Smit, H.A. and Van Pelt, W., 2013. Infectious disease burden related to child day care in the Netherlands. Pediatric Infectious Disease Journal 32: e334-340. https://doi.org/10.1097/INF.0b013e318290601e

Eurydice Report, 2019. Key data on early childhood education and care in Europe, 2019. Available at: https://op.europa.eu/s/ofpk

Everard, A., Lazarevic, V., Gaïa, N., Johansson, M., Ståhlman, M., Backhed, F., Delzenne, N.M., Schrenzel, J., François, P. and Cani, P.D., 2014. Microbiome of prebiotic-treated mice reveals novel targets involved in host response during obesity. ISME Journal 8: 2116-2130. https://doi.org/10.1038/ismej.2014.45 
Forbes, J.D., Van Domselaar, G. and Bernstein, C.N., 2016. The gut microbiota in immune-mediated inflammatory diseases. Frontiers in Microbiology 7: 1081-1081. https://doi.org/10.3389/ fmicb.2016.01081

Hakim, H., Dallas, R., Wolf, J., Tang, L., Schultz-Cherry, S., Darling, V., Johnson, C., Karlsson, E.A., Chang, T.-C., Jeha, S., Pui, C.-H., Sun, Y., Pounds, S., Hayden, R.T., Tuomanen, E. and Rosch, J.W., 2018. Gut microbiome composition predicts infection risk during chemotherapy in children with acute lymphoblastic leukemia. Clinical Infectious Diseases 67: 541-548. https://doi.org/10.1093/ $\mathrm{cid} /$ ciy 153

Hill, C., Guarner, F., Reid, G., Gibson, G.R., Merenstein, D.J., Pot, B., Morelli, L., Canani, R.B., Flint, H.J., Salminen, S., Calder, P.C. and Sanders, M.E., 2014. The International Scientific Association for Probiotics and Prebiotics consensus statement on the scope and appropriate use of the term probiotic. Nature Reviews Gastroenterology and Hepatology 11: 506-514. https://doi. org/10.1038/nrgastro.2014.66

Hojsak, I., 2017. Probiotics in children: what is the evidence? Pediatric Gastroenterology, Hepatology and Nutrition 20: 139-146. https:// doi.org/10.5223/pghn.2017.20.3.139

Hong, H.A., Khaneja, R., Tam, N.M., Cazzato, A., Tan, S., Urdaci, M., Brisson, A., Gasbarrini, A., Barnes, I. and Cutting, S.M., 2009. Bacillus subtilis isolated from the human gastrointestinal tract. Research in Microbiology 160: 134-143. https://doi.org/10.1016/j. resmic.2008.11.002

Horosheva, T.V., Sorokulova, I. and Vodyanoy, V., 2014. Efficacy of Bacillus probiotics in prevention of antibiotic-associated diarrhoea: a randomized, double-blind, placebo-controlled clinical trial. JMM Case Reports 1: 4036. https://doi.org/10.1099/jmmcr.0.004.036

Inatsu, Y., Nakamura, N., Yuriko, Y., Fushimi, T., Watanasiritum, L. and Kawamoto, S., 2006. Characterization of Bacillus subtilis strains in Thua nao, a traditional fermented soybean food in northern Thailand. Letters in Applied Microbiology 43: 237-242. https:// doi.org/10.1111/j.1472-765X.2006.01966.x

Labellarte, G., Cooper, S. and Maher, M., 2015. Tolerance and efficacy of a probiotic supplement delivered in capsule form. FASEB Journal 29: 924-933. https://doi.org/10.1096/fasebj.29.1_supplement.924.33

Lakshmi, S.G., Jayanthi, N., Saravanan, M. and Ratna, M.S., 2017. Safety assesment of Bacillus clausii UBBC07, a spore forming probiotic. Toxicology Report 4: 62-71. https://doi.org/10.1016/j. toxrep.2016.12.004

Laursen, M.F., Laursen, R.P., Larnkjaer, A., Michaelsen, K.F., Bahl, M.I. and Licht, T.R., 2017. Administration of two probiotic strains during early childhood does not affect the endogenous gut microbiota composition despite probiotic proliferation. BMC Microbiology 17: 175. https://doi.org/10.1186/s12866-017-1090-7

Laursen, R.P. and Hojsak, I., 2018. Probiotics for respiratory tract infections in children attending day care centers-a systematic review. European Journal of Pediatrics 177: 979-994. https://doi. org/10.1007/s00431-018-3167-1

Lefevre, M., Racedo, S.M., Denayrolles, M., Ripert, G., Desfougeres, T., Lobach, A.R., Simon, R., Pelerin, F., Justen, P. and Urdaci, M.C., 2017. Safety assessment of Bacillus subtilis CU1 for use as a probiotic in humans. Regulatory Toxicology and Pharmacology 83: 54-65. https://doi.org/10.1016/j.yrtph.2016.11.010
Louhiala, J.N., Ruotsalainen, R. and Jaakkola, J.J.K., 1997. Day-care centers and diarrhea: a public health perspective. Journal of Pediatrics 131: 476-479.

Love, M.I., Huber, W. and Anders, S., 2014. Moderated estimation of fold change and dispersion for RNA-seq data with DESeq2. Genome Biology 15: 550. https://doi.org/10.1186/s13059-014-0550-8

McMurdie, P.J. and Holmes, S., 2013. Phyloseq: an R package for reproducible interactive analysis and graphics of microbiome census data. PLoS ONE 8: e61217. https://doi.org/10.1371/journal. pone. 0061217

Nafstad, P., Hagen, J.A., Oie, L., Megnus, P., Jaakkola, J.J.K., 1999. Day care centres and respiratory health. Pediatrics 103: 753-758. https:// doi.org/10.1542/peds.103.4.753

Pallmann, P., Schaarschmidt, F., Hothorn, L.A., Fischer, C., Nacke, H., Priesnitz, K.U. and Schork, N.J., 2012. Assessing group differences in biodiversity by simultaneously testing a user-defined selection of diversity indices. Molecular Ecology Resources 12: 1068-1078. https://doi.org/10.1111/1755-0998.12004

Parada Venegas, D., De la Fuente, M.K., Landskron, G., Gonzalez, M.J., Quera, R., Dijkstra, G., Harmsen, H.J.M., Faber, K.N. and Hermoso, M.A., 2019. Short chain fatty acids (SCFAs)-mediated gut epithelial and immune regulation and its relevance for inflammatory bowel diseases. Frontiers in Immunology 10: 277. https://doi.org/10.3389/ fimmu.2019.00277

Paytuví, A., Battista, E., Scippacercola, F., Aiese Cigliano, R. and Sanseverino, W., 2019. GAIA: an integrated metagenomics suite. bioRxiv: 804690 . https://doi.org/10.1101/804690

Ray, P., Sanchez, C., O'Sullivan, D.J. and McKay, L.L., 2000. Classification of a bacterial isolate, from pozol, exhibiting antimicrobial activity against several gram-positive and gram-negative bacteria, yeasts, and molds. Journal of Food Protection 63: 1123-1132. https://doi. org/10.4315/0362-028x-63.8.1123

Reese, A.T. and Dunn, R.R., 2018. Drivers of microbiome biodiversity: a review of general rules, feces, and ignorance. mBio 9: e01294-18. https://doi.org/10.1128/mBio.01294-18

Shen, X., Miao, J., Wan, Q., Wang, S., Li, M., Pu, F., Wang, G., Qian, W., Yu, Q., Marotta, F. and He, F., 2018. Possible correlation between gut microbiota and immunity among healthy middle-aged and elderly people in southwest China. Gut Pathogens 10: 4. https:// doi.org/10.1186/s13099-018-0231-3

Strong, W.L., 2016. Biased richness and evenness relationships within Shannon-Wiener index values. Ecological Indicators 67: 703-713. https://doi.org/10.1016/j.ecolind.2016.03.043

Suva, M., Sureja, V. and Kheni, D., 2016. Novel insight on probiotic Bacillus subtilis: mechanism of action and clinical applications. Journal of Current Research in Scientific Medicine 2: 65-72. https:// doi.org/10.4103/2455-3069.198381

Toohey, J.C., Townsend, J.R., Johnson, S.B., Toy, A.M., Vantrease, W.C., Bender, D., Crimi, C.C., Stowers, K.L., Ruiz, M.D., VanDusseldorp, T.A., Feito, Y. and Mangine, G.T., in press. Effects of probiotic (Bacillus subtilis) supplementation during offseason resistance training in female division I athletes. Journal of Strength and Conditioning Research. https://doi.org/10.1519/ jsc.0000000000002675 
Van der Wielen, G.C., Gothefors, L., Huelsse, C., Huet, F., Littmann, M., Maxwell, M., Talayero, J.M.P., Todd, P., Vila, M.T., Cantarutti, L., Van Damme, P. and the REVEAL Study Group, 2010. Impact of community-acquired paediatric rotavirus gastroenteritis on family life: data from the REVEAL study. BMC Family Practice 11: 22-27.

Wallace, B., 2009. Clinical use of probiotics in the pediatric population. Nutrition in Clinical Practice 24: 50-59. https://doi. org/10.1177/0884533608329298

Wang, J. and Fung, D.Y.C., 1996. Alkaline-fermented foods: a review with emphasis on Pidan fermentation. Critical Reviews in Microbiology 22: 101-138. https://doi.org/10.3109/10408419609106457
Xiao, L., Gong, C., Ding, Y., Ding, G., Xu, X., Deng, C., Ze, X., Malard, P. and Ben, X., 2019. Probiotics maintain intestinal secretory immunoglobulin A levels in healthy formula-fed infants: a randomised, double-blind, placebo-controlled study. Beneficial Microbes 10: 729-739. https://doi.org/10.3920/bm2019.0025

Zeng, H., Ishaq, S.L., Zhao, F.-Q. and Wright, A.-D.G., 2016. Colonic inflammation accompanies an increase of $\beta$-catenin signaling and Lachnospiraceae/Streptococcaceae bacteria in the hind gut of highfat diet-fed mice. Journal of Nutritional Biochemistry 35: 30-36. https://doi.org/10.1016/j.jnutbio.2016.05.015

Zhou, Y. and Zhi, F., 2016. Lower level of Bacteroides in the gut microbiota is associated with inflammatory bowel disease: a metaanalysis. BioMed Research International 2016: 5828959. https://doi. org/10.1155/2016/5828959 